\title{
The Transition from Comprehensive to Analytical Characteristics of English Language
}

\author{
Xiaqing Li \\ Department of Minority Languages and Literatures, Minzu University of China, Beijing, China
}

\begin{abstract}
English belongs to the Indo-European language family. It is a language that achieves meaning expression through its own form of inflection, focusing on form, and it is one kind of comprehensive language. Analytical language expresses grammatical meaning through function words, word order, and so on. With the development of language, English has a tendency to develop from comprehensive language to analytical language. In this study, it explains the transitional characteristics of English language from comprehensive characteristics to analytical characteristics from following aspects: reduces or even disappears of English morphological change; the ability of English vocabulary meaning expansion; the multi-use of function words; the powerful grammatical function of the article; the fusion of English and Chinese language; the Chinese loanword in English; the Syntactical phenomenon of English and Chinese language, the complexity of English word formation, the theme-highlight sentence pattern.
\end{abstract}

Index Terms -English, comprehensive characteristics, analytical characteristics, inflection

\section{INTRODUCTION}

Comprehensive language means that the language expresses grammatical meaning (grid, number, time) mainly through the morphological change of the word itself. The so-called analytical language means that the language expresses the grammatical relationship in this language is mainly not through the form of the word itself, but expresses through the function word, and word order. (Yang Siyuan, 2009) Although English is a comprehensive language, the development of English has gone through the process from Old English to Middle English to modern English. In the process of development and evolution, the inflection of English itself has gradually decreased. In modern English, some of the inflections of the original words have disappeared. In addition, the comprehensive language of English shows some of the same characteristics as the analytical language of Chinese in some respects. The following describes the analytical characteristics of English from some aspects.

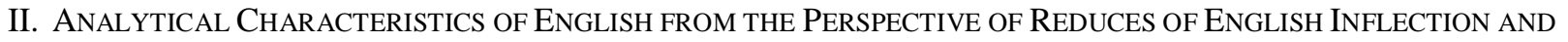 MORPHOLOGICAL CHANGE}

Both Old English and modern English have great differences in writing, pronunciation, vocabulary and grammar. They are generally considered to belong to the SOV-type subject-object structure. In addition, the inflections of Old English words are very rich, Nouns have positive, negative, neutral, and various "grid" changes, from the following grammatical changes in the first person of Old English. We can see this feature, of course, there are some inflections in other aspects such as plurals and suffixes. Here we only list the changes in the first person singular number, as follows:

\begin{tabular}{|l|l|l|l|l|}
\multicolumn{2}{l}{ THE CHANGES IN THE FIRST PERSON SINGULAR NUMBER } \\
\begin{tabular}{|l|l|l|l|l|}
\hline & Nominative & Objective & Genitive & Dative \\
\hline Positive & min & Mine & Mires & minum \\
\hline Neutral & min & Min & mines & minum \\
\hline Negative & minu & mine & minre & minre \\
\hline
\end{tabular}
\end{tabular}

With the conquest of the British in the eleventh century, Old English gradually transitioned to Middle English. Compared with Old English, Middle English has gradually reduced the inflection of words. By the fifteenth century, Middle English had completely become the subject-verb-object structure of SVO, and the development of modern English, SVO's word order has basically stabilized. In addition to the irregular changes in modern English and the "lattice" changes of a few pronouns, the external inflections have only the following five aspects: First, in the current tense, the nouns are followed by the suffix s or es, indicating single and plural forms of nouns.. When the third person singular is the subject in the sentence, the predicate verb is also suffixed with s or es. Second, after the verb, the suffix ed indicates the past tense and the past participle. Third, after the verb, the suffix ing indicates the present participle and gerund of the verb. Fourth, add the suffix er after the adjective or adverb to indicate their comparison level. Fifth, add the affix est after the adjective or adverb to indicate the highest level of the adjective or adverb. It can be said that from the development of old English to modern English, the grammatical form marks are continuously decreasing, and the inflection changes gradually disappear. (Ding Zhibin \& Shi Hongmei, 2011) The disappearance of inflection and the fixation of English syntactic forms are almost simultaneous. The external inflection change of modern English is 
simplified into the above several kinds. The simplification or disappearance of grammatical form marks also accelerates the process of word order fixation. The SVO structure of modern English gradually replaces the old English word order based on SOV structure, which is in line with the nature of human thinking. The logical order is also a manifestation of the analytical characteristics of modern English.

\section{Analytical Characteristics of English from the Perspective of the Strong Ability OF EnGLiSh VOCABULARY MEANING EXPANSION}

The ability to expand the meaning of the term is related to the analytical strength of the language. Most of the time: the analytical characteristics of language are very obvious, and meaning expansion ability of the language is strong (Dai Qingxia, 2017). The polysemy of word meaning is a common phenomenon in human language. In almost all languages, polysemy accounts for the main body of vocabulary. The proportion of monosyllabic words is far less than that of polysemous words. English is no exception. There are many polysemy phenomenon in English.. (Hu Yi, 2012) Especially the polysemy phenomenon of the words related to the body and feelings and daily life are very common in English. From the polysemy of these words, we can see that the expansion ability of English word meanings is very strong.

Here are some examples to illustrate the expansion of the meaning of some words in English. For example, the word "hand", the basic meaning is the hand, and it can be extended to the furgative meaning of "on hand" or "at hand"; it can extended to the analogy of an old hand, meaning is the veteran; it also can extend to the metaphorical meaning "at first hand". For example, the word "eye", its basic meaning is the eye, extended to the eye of a storm, refers to the eye, extended to the metaphorical eyes and ears, and an eye for something. The above two examples illustrate the polysemy of words in English that represent body organs. The word "bitter" which expresses the feeling of body in English, its basic meaning is "bitter", which is extended to the meaning of "bitter pill", which means bitter fruit. Expanding to "bitter experience" is a painful experience. Expanding to "bitter tears" is the meaning of painful tears. Expanding to "bitter smile" means bitterness. For example, the word "bed" related to daily life, its basic meaning is the bed, which can be extended to the meaning of "seedbed", referring to the seedbed. Expanded to "a bed of rock", and it refers to the deposit. Expanded to metaphorical "hotbed", and it refers to the hotbed. Expanded to "drapes over a bed", referring to the bed.

There are many more polysemy words about vocabulary about body, feeling, and daily life in English. I will not list them here. From the polysemy of these words, it can be seen that the basic meaning of English vocabulary can be extended by many extensions and metaphors, and English has a strong ability to expand. This is an analytical feature of English.

\section{Analytical Characteristics of English from the Perspective of Multi-Use of Function Words And ITS POWERFUL GRAMMATICAL FUNCTION}

In language, a function word refers to a word that has no real meaning, and cannot independently assume elements of sentence, such as a be-verb, or a preposition. Another feature of the word is that there is no change in the form. Function words have the grammatical meaning of connecting or attaching various types of content words. In English, function words include prepositions and conjunctions. But now with the constant change of language, some function words are used frequently in English, and can express some practical meanings according to different usages, reflecting the analytical characteristics of English language. The following, the author explains the analytical characteristics of English language from different aspects with prepositions and conjunctions of English function words.

Seeing the analytical characteristics of English from the use of prepositions. Prepositions, also known as "prepositions (qian zhi ci)", indicate the relationship between nouns, pronouns, and other words in a sentence. They cannot be used as separate part in a sentence. Prepositions are generally used in front of nouns or pronouns to indicate the relationship between the sentence and other components in the sentence. The noun or pronoun after the preposition is called the preposition object. The preposition and the prepositional object together form a prepositional phrase. The status of interlanguage in modern English is very important. We must not underestimate this small role, nor can we ignore its role.

There are some following prepositions in English intermediaries, and these prepositions represent different concepts. Common prepositions that represent time are at, on, in, and so on. For example, on Monday is "on" Monday. Common prepositions during the presentation period are during, for, over, within, throughout, from and to. For example, during his childhood. Prepositions that represent other time concepts are before, after, since, until, till, between, up to, etc. The prepositions indicating orientation are at, in, on, to, for, above, over, below, under, in front of, in the front of, behind, beside. It can be seen from the above that some prepositions that express time can also indicate orientation. Indicating "go", and when "go" is used as an adverbial, an English preposition can indicate an action that is "going". Example: The house is on fire! The house is on fire! Indicating the reason, commonly used prepositions are "for" and so on. Common prepositions that express "methods", and "means" are by, with, through, etc. Common prepositions that represent quantities are about (around), over, and so on. 
In addition, intransitive verbs cannot directly follow with the object, and have a passive voice. However, after matching with prepositions, intransitive verbs can not only follow with objects, but also have a passive voice. From the above examples, it can be seen that although the preposition is a small word class, most of the words are small and spelled simply. However, the frequency of use is quite high, and it is complicated and varied in the English conventional structure. Whether they are prepositions or prepositional phrases, they are powerful in English sentences. They express grammatical meaning through different relationships and positions in other English sentences. This is also an analytical feature of English

Seeing the analytical characteristics of English from the multi-use of conjunctions. A conjunction is a kind of function word. It can't be a sentence component independently, but only it plays a role of connecting words and words, phrases and phrases, and sentences and sentences. Conjunctions can be divided into two main categories: juxtaposed conjunctions and subordinate conjunctions. The multi-use of conjunctions also represents the multi-purpose use of function words. Following it mainly explains the multi-use phenomenon of juxtaposed conjunctions.

Parallel conjunctions are used to connect parallel words, phrases, and clauses. For example, the word "and" has the following usages, connecting two parallel verbs, such as: They sat down and talked about something; connecting two parallel verb infinitives, the second infinitive often omits to, such as: They started to dance and sing; it can be used in conjunction with imperative sentences or noun phrases, such as: Make up your mind, and you'll get the chance. When expressing the whole or referring to the same person, the predicate verb is singular; when the "and" is connected to the singular noun, predicates are also singular when they are modified by the words "each", "every", and "no". Such as: The bread and butter is really delicious; when connecting two words with the same meaning, it means "gradual", or "strengthen the tone", such as: read it again and again. The conjunction "both..and", such as: Both he and she play the piano.

About the conjunction "neither...nor" predicate verbs adopt the principle of proximity, which is consistent with the words after the word "nor". Such as: Neither you nor he is to blame. About the example conjunction "not only, but also", such as: She plays not only the piano, but (also) the guitar. When the conjunction "not only, but also" associates two clauses, one clause must be flipped because of the negative word not. Not only does he like reading stories, but also he can even write some. The conjunction "as well as", the predicate verb is not affected by the components followed by "as well as". Such as: The teacher, as well as the students, is interested in the activity. The conjunction "or" indicates the choice of relationship, or otherwise, such as: Which do you prefer, coffee or juice? Hurry up, or you'll be late for The class. About the conjunction "either...on" predicate verb adopts the principle of proximity, such as: Either you or I am right. About the example of the conjunction "or else/otherwise" such as: Be silent, or else you will be kicked out. Another example is "I am tired, otherwise, I would play. There is a conjunction "but" that indicates a turning or contrasting relationship, such as: He is rich but unhappy. Following are another two examples about two conjunctions "while", "however", indicating a turning or contrasting relationship. such as: Some people love cats, while others hate them. About the example of the conjunction "yet", such as: Some said She would be late, yet she arrived on time. About the example of the conjunction "however" such as: She does not like him, however, I like him. About the example of the conjunction "not, but" such as: It's not my book, but is his book. The prepositions that express causality are for, so, therefore, then, etc. When the word "for" is used as the parallel conjunction, the reason for the preceding sentence is added. Only sentences and sentences can be connected, usually not at the beginning of the sentence. Such as: He is absent today, for he is ill. So, the word "therefore" means therefore, such as: He hurt his leg, so he couldn't play in the game. I think, therefore I am. About "then" such as: Run fast, then he won't catch you.

From the above conjunctions and their related examples and usages, the use of conjunctions is also a lot in English, with irreplaceable status. It expresses grammatical meaning not by changing their own form, but by the relationship between them and other elements in the sentence, and in most cases it can determine the form of other components in the sentence, such as predicate. Different conjunctions represent different relationships whether it is a parallel relationship, a transitional relationship, a causal relationship, or a comparative relationship, a conjunction has a very important position in constructing English sentences and paragraphs. This is also a major manifestation of the gradual transition of modern English to analytical language.

\section{Analytical Characteristics of English from the Perspective of the Compatibility of ENGLish AND CHINESE}

Some words in English can express Chinese vocabulary. Although English and Chinese are influenced by different cultural customs and different language features, they have striking similarities in linguistic form and stylistic image. It is becoming more and more common on phenomenon of compatibility of English and Chinese, manifested in all levels of language. Among them, the compatibility of lexical level is the most prominent. One is the transliteration of words produced by English-Chinese compatibility. When it is introduced in English vocabulary, there is no corresponding vocabulary expression in Chinese, so new words are invented by transliteration, which are both simple and exotic, such as "nylon", and "coffee". These two words are transliterated, and such words are also very prominent in the food culture, such as: salad, pudding, hamburger, chocolate, coca cola, whisky, toast, etc., there are many other words in the field, such as: ballet, salon, model, copy, AIDS, clone, gene, vitamin, fee, microphone, cool, etc. From these transliterated words, it can be seen that the pronunciation and the meaning of words are very similar. We can distinguish the 
corresponding Chinese words and meanings from the pronunciation of most words. It is very similar with characteristics of using forms to express meaning of Chinese. Although we say that Chinese is a language of using forms to express meanings, English is a language of using sound to express meaning, but there are many meanings of words in English that cannot be recognized by pronunciation, and these transliteration words of English and Chinese are different, and they can be known corresponding Chinese words from their pronunciation, further, its meaning is understand, reflecting the analytical characteristics of English. In addition, the compatibility of English and Chinese vocabulary is also reflected in vocabulary borrowing and English word formation. The analytical characteristics of English are explained as the following two aspects.

Seeing the analytical characteristics of English from the Chinese loanwords in English. Most languages in the world have the phenomenon of borrowing words from other languages. (Wang Zhiqiang, 2012) The borrowing of vocabulary is also the reason of vocabulary compatibility. As the exchanges between countries become more frequent, the culture also learns from each other. As a manifestation of culture, language also appears to be borrowed from each other under the influence of frequent cultural exchanges. In addition, with the development and progress of society, many new things appear, and their own English vocabulary can not meet the needs of expressing new things, so in English many Chinese words are borrowed, such as: "fengshui", "qigong", "confucianism”, "Taoism", "kowtow"(skull), "lose face" (shame face), "paper tiger", "ying", "yang", "kung-fu", "Tai chi", "silk", "kooli" (cool), "yoga" from these new words, it can be seen that these words are very similar to the pronunciation of the corresponding words in Chinese. From the pronunciation of these words, we can know the corresponding words and their meanings, and the characteristics of using the pronunciation to express meaning are very obvious. Moreover, these loan words are created according to the pronunciation of the corresponding Chinese, and are created by a special new English vocabulary to represent the Chinese corresponding words, instead of being created by inflection on the basis of English words. This is the same as the way of creating Chinese, which is also the embodiment of the analytical features of English.

Seeing the analytical characteristics of English from the complexity of word formation. There are many syllables in the English word itself, and this trend is more obvious due to the large number of compound words. At the same time, with the development of society and the emergence of new things, new concepts and new ideas are emerging, and people's thinking is becoming more and more refined. In order to accurately reflect these changes, the internal structure of English vocabulary has to be complicated, and only through the word inflection, and this way is no longer sufficient for creating English words. The complexity of English word formation is gradually similar to the complexity of Chinese word formation. Similar to the word formation of "half-ton couple" and "big belly economy" in Chinese, there are also complex words such as "talk-show campaign" and "jerry-built" project in English. From the three word formation methods of English, such as compound word formation, analog word formation, and abbreviations, we can see the similarity between English and Chinese word formation, and we can see that the English language has the same analytical characteristics as Chinese.

The compound word formation in English is also the main way of word formation, and the number of compound words in English is also quite large. The compound method refers to the method of arranging two or more words into a new word in a certain word order. In English, the frequency of compound word formation is also very high. (Yu Juanjuan \& Zhang Yanling, 2009) In recent years, compound words in English such as: "petrodollar", "merry killing", "serial time sharing system", and "open heart surgery". We can see from these words composed of compound word formation that these words are not formed by inflection in the form of the word itself, but by combining two or more words. we can't see any characteristics of inflection in these new English words.

In the process of English word formation, the analogy word formation method is also the main word formation method. The analogy method refers to the use of a similar word as a model, semantically associating analogies, replacing one of the morphemes, and imitating new words corresponding to or similar. In English, this analogy is also common, such as "blue-collar" and "white-collar" analogy "pink-collar", "gray-collar", and the "gold-collar". We can see from these words composed of analogical word formation that these words are formed by analogy on the basis of existing similar words. New words are composed of two separate words, rather than through the morphological changes of words. The resulting morphological changes in English words are not seen in these words.

Abbreviation is also one of the most important means of word formation in modern languages. There are also many acronyms in English, such as: "AI" (artificial intelligence), "URL" (uniform resource locator), and "SARS". These word is formed by processing the abbreviated initials of original complete words, rather than by inflection of the words. It can be seen that although English is not an ideographic language, most words cannot be expanded or reduced like Chinese, but with very active acronym generation capabilities.

In general, whether compound words, analog words, or abbreviations, they form new words by rearranging or abbreviating two or more words to express new things corresponding to Chinese. From these words, we can't see any traces of inflection of English word form, but similar to Chinese word formation, and these words have strong ideographic functions, reflecting the analytical characteristics of English.

\section{CONCLUSION}

In summary, from old English to modern English, the change of inflectional form is reduced; some words related to body, feeling, and daily life in English can be extended from their basic meanings to extended meanings and metaphors. 
These words embody the similar meaning extending features of English words and Chinese. It has strong ability to expand the meaning; English function words include conjunctions, and prepositions. These words are used in English and they have the powerful grammatical functions. English function words are no longer just as an aid way of sentence, and more is to express the actual meaning; from the transliteration words formed by the compatibility of English and Chinese language, it can be seen that the characteristics of using sound to express meaning in English are very obvious; from the Chinese loanwords in English and the complexity of English word formation, It can be seen that the related words and the complex composition of the words do not reflect the inflectional characteristics of the English words; for the purpose of special discourse or rhetoric, there are also many cases in which the subject-prominent English language it has many cases that theme-prominent sentences emerge. It reflects the analytical characteristics of modern English and shows that modern English has a tendency to develop from comprehensive language to analytical language.

From the development of the English language itself, we find that modern English has a tendency to reduce the form of inflection in the form of words and syllables, which reflects the analytical characteristics of English. From the following similarities of comprehensive English and analytical Chinese: the ability to expand the meaning, the multiuse of function words, the complexity of word formation, and the prominent features of the theme reflect the analytical characteristics of the English language. From these aspects, we realize that the comprehensive language of English has a tendency to develop analytical language, with the gradual disappearance of inflection of English and the economic principle of language, perhaps in the near future, English will become an analytical language completely. At present, as the language changes, researchers need to look at the language of English with a dynamic development, and it will provide guidance for researchers to understand and analyze English language. Further, it can also provide guidance for English teaching.

\section{REFERENCES}

[1] Dai Qingxia. J. (2017). Re-exploration of Characteristics of Chinese. Minority Languages of China. 2, 3-10.

[2] Ding Zhibing \& Shi Hongmei. J. (2011) Typology Characteristics in English Grammaticalization Evolution. Foreign Language Teaching (Vol.32)2,19-22.

[3] Hu Yi. J. (2012). Taking Vocabulary as an Example to Discuss English Learning Strategies from the Perspective of Linguistic Typology. Higher Education Forum.5, 125-128.

[4] Ou Wenting. J. (2014). Number-Category Analysis of Zhuang Language and English in the Framework of Linguistic Typology. Journal of Qinzhou University(Vol.29).12,56-58.

[5] Wang Zhiqiang. (2012). The Each Other Between English and Chinese Load--Words Influence: The Period From 1978 to the Present. Huainan normal university. https://wenku.baidu.com/view/aa806bab0b1c59eef9c7b473.html (accessed 13/8/2018).

[6] Yang Lianrui. D. (2008). Topic Prominence in Typological Interlanguage Development of Chinese Students' English. Shanghai International Studies University.

[7] Yang Siyuan. (2009). The Differences of Chinese Characters and English Letters. http://www.docin.com/p-359909790.html (accessed 13/8/2018).

[8] Yu Juanjuan \& Zhang Yanling. J. (2009). The Common Characters of Chinese and English in formation of New Words. Journal of Suzhou University( Vol.24).4, 57-60.

Xiaqing $\mathbf{L i}$ is a Doctor degree candidate in the Department of Minority Languages and Literature at Minzu University of China in capital city Beijing, in China, where she is pursuing her Doctor's degree in linguistics and applied linguistics. Her research interests include computational linguistics, and applied linguistics. 